Portland State University

PDXScholar

Mechanical and Materials Engineering Faculty

Publications and Presentations

2020

\title{
Impact of Cognitive Tasks on CO2 and Isoprene Emissions from Humans
}

\author{
Elliott T. Gall \\ Portland State University, gall@pdx.edu \\ Asit Kumar Mishra \\ Berkeley Education Alliance for Research in Singapore \\ Jiayu Li \\ Berkeley Education Alliance for Research in Singapore \\ Stefano Schiavon \\ University of California, Berkeley \\ Aurelie Laguerre \\ Portland State University, aurelie.prot@pdx.edu
}

Follow this and additional works at: https://pdxscholar.library.pdx.edu/mengin_fac

Part of the Engineering Commons

Let us know how access to this document benefits you.

\section{Citation Details}

Gall, E. T., Mishra, A. K., Li, J., Schiavon, S., \& Laguerre, A. (2020). Impact of Cognitive Tasks on CO2 and Isoprene Emissions from Humans. Environmental Science \& Technology.

This Pre-Print is brought to you for free and open access. It has been accepted for inclusion in Mechanical and Materials Engineering Faculty Publications and Presentations by an authorized administrator of PDXScholar. Please contact us if we can make this document more accessible: pdxscholar@pdx.edu. 


\section{Impact of cognitive tasks on $\mathrm{CO}_{2}$ and isoprene 2 emissions from humans}

3 Elliott T. Gall ${ }^{1, *}$, Asit Kumar Mishra ${ }^{2}$, Jiayu Li ${ }^{2}$, Stefano Schiavon ${ }^{3}$, Aurélie Laguerre ${ }^{1}$

$4{ }^{1}$ Department of Mechanical and Materials Engineering, Portland State University, Portland, OR, 5 USA.

$6 \quad{ }^{2}$ Berkeley Education Alliance for Research in Singapore, Singapore

$7 \quad{ }^{3}$ Center for the Built Environment, University of California, Berkeley, CA, USA

8 *Corresponding author:

91930 SW $4^{\text {th }}$ Ave, Portland, OR 97201, Suite 400, Department of Mechanical and Materials

10 Engineering, Portland State University, Portland, OR, USA Email: gall@ pdx.edu

\section{Abstract}

The human body emits a wide range of chemicals, including $\mathrm{CO}_{2}$ and isoprene. To examine the impact of cognitive tasks on human emission rates of $\mathrm{CO}_{2}$ and isoprene, we conducted an across subjects, counterbalanced study in a controlled chamber involving 16 adults. The chamber replicated an office environment. In groups of four, participants engaged in 30 minutes each of cognitive tasks (stressed activity) and watching nature documentaries (relaxed activity). Measured biomarkers indicated higher stress levels were achieved during the stressed activity. Per-person $\mathrm{CO}_{2}$ emission rates were greater for stressed than relaxed activity $(30.3 \pm 2.1$ vs. $27.0 \pm 1.7 \mathrm{~g} / \mathrm{h} / \mathrm{p}, p=0.0044$, mean \pm standard deviation $)$. Isoprene emission rates were also elevated under stressed vs. relaxed activity $(154 \pm 25 \mu \mathrm{g} / \mathrm{h} / \mathrm{p}$ vs. $116 \pm 20$ $\mu \mathrm{g} / \mathrm{h} / \mathrm{p}, p=0.041)$. Chamber temperature was held constant at $26.2 \pm 0.49{ }^{\circ} \mathrm{C}$; incidental variation in temperature did not explain variance in emission rates. Isoprene emission rates increased linearly with salivary-alpha amylase levels $\left(r^{2}=0.6, p=0.02\right)$. These results imply the possibility of considering cognitive tasks when determining building ventilation rates. They also present the possibility of monitoring indicators of cognitive tasks of occupants through measurement of air quality.

Keywords: human emissions; bioeffluents; stress biomarkers; $\mathrm{CO}_{2}$; isoprene

\section{Introduction}

Human chemical emissions of gaseous or particle-phase compounds associated with human metabolism include carbon dioxide, ${ }^{1}$ volatile organic compounds (VOCs), ${ }^{2}$ and bioaerosols. ${ }^{3,4}$ These compounds, emitted via human breath and skin, impact indoor air chemistry and contribute to degraded indoor air quality. ${ }^{5,6}$ Human chemical emissions, often indicated by proxy measurement of carbon dioxide $\left(\mathrm{CO}_{2}\right)$, are also a driver of the need to ventilate buildings, with prominent building ventilation standards like ASHRAE 62.1 and EN 
16798 based on removing odorous human chemical emissions from the indoor environment. ${ }^{7}$

Exposure to human chemical emissions may also impact human cognition. ${ }^{8,9}$ Studies have implicated exposure to pure elevated $\mathrm{CO}_{2}$ as impairing human cognition, ${ }^{10,11}$ though there also exist studies that show no effect of $\mathrm{CO}_{2}$ itself on cognition. ${ }^{12,13}$ However, it is consistently shown that reduced outdoor air ventilation, leading to higher indoor concentration of human chemical emissions, is responsible for observed decrements in cognition or environment perception. ${ }^{14-16}$

A variety of factors beyond ventilation rate and occupant density impact the level of human chemical emissions in a given indoor space. There are methods for predicting the $\mathrm{CO}_{2}$ generation rate per-occupant, ${ }^{17,18}$ and they consider body surface area, body composition, metabolic rate (related to type of activity), air temperature, and a respiratory quotient, which is largely a function of diet. ${ }^{19}$ There are many studies in the literature quantifying type and quantity of emissions of endogenous and exogenous volatile organic compounds, ${ }^{20-23}$ often in pursuit of understanding the human "volatilome" for diagnosis of disease. ${ }^{24}$ Enabled by analytical methods that can measure VOCs with high time resolution, studies have estimated average, per-person emission rates of carbon dioxide and VOCs in a variety of field settings including classrooms, ${ }^{2,25,26}$ a museum, ${ }^{27}$ and a movie theater ${ }^{28}$; these studies have substantially expanded our knowledge of the magnitudes and type of human chemical emissions. However, comparatively fewer studies investigate factors in healthy humans that may influence human chemical emissions, including traits like age, sex, or smoking status, ${ }^{29-31}$ and their biochemical mechanisms. ${ }^{32}$ Recent studies, conducted in controlled chambers, measured human emissions under varying "human factors" including type of clothing worn and age, as well as environmental factors, including temperature. ${ }^{33,34}$ 
59 or understood. In a 1975 field study of human bioeffluent emissions, Wang et al. ${ }^{35}$ measured

60 volatile emissions from students in a University classroom used for lectures and examinations.

61 They found twelve organic compounds were elevated during lecture periods, and estimate per-

62 person production rates. In that study, the researchers identified and separated examination

63 periods as a condition where occupants experienced increased stress relative to lecture. They

64 reported a $43 \%$ increase in $\mathrm{CO}_{2}$ emission rate when comparing examination to lecture

65 conditions; the authors suggest that this was due to elevated metabolism. More recently,

66 Williams et al. ${ }^{36}$, investigated the role of audiovisual stimuli to influence human chemical

67 emissions using a high time-resolution proton transfer reaction - time of flight - mass

68 spectrometer installed in the heating, ventilation and air-conditioning system of a movie

69 theater. By annotating films with scene content labels, the researchers ascertained whether a

70 scene label could predict variation of the measured human chemical emissions. Carbon

71 dioxide and isoprene exhibited high correlations with scene labels, indicating emissions of

72 these compounds may be associated with biochemical pathways induced by emotional

73 responses to the movie scene. Isoprene emissions and variability have also been studied as a

74 potential marker for age-appropriateness of film content, ${ }^{37}$ and may serve as an indicator for

75 several metabolic processes in the human body, including lipid metabolism and physiological

76 state. $^{38}$

77 Better understanding of factors influencing human chemical emission, including

78 stimuli such as cognitive tasks and audiovisual experience, will advance our understanding of

79 metabolic and biochemical pathways that may be initiated as a result of cognitive processes.

80 Such knowledge could be applied to improve ventilation strategies in buildings by, for 
81 example, tailoring ventilation strategies to the psychological experience and/or commitments

82 of the occupants. Conversely, monitoring levels of human chemical emissions in buildings could be used to gauge occupant cognitive engagement. To date, studies advancing our knowledge of factors influencing human chemical emissions have generally occurred in uncontrolled field environments (movie theaters, classrooms, museums). We use a state-ofthe-art controlled test chamber to engage sixteen subjects in an across subjects, counterbalanced study, to explore how cognitive tasks may influence human chemical emission rates of isoprene and carbon dioxide. To our knowledge, this is the first controlled study of the effect of cognitive tasks on human chemical emission rates.

\section{Methods and materials}

\subsection{Test chamber and subjects} cognitive tasks on $\mathrm{CO}_{2}$ and isoprene emitted by humans. The test chamber is located within

We conducted studies in a room-scale test chamber to measure the influence of the Singapore-Berkeley Building Efficiency for Sustainability in the Tropics facility (Figure S1, Supporting Information). The test bed consists of four climatic chambers, each $5.6 \times 4.3 \times 2.7 \mathrm{~m}$. Our tests were conducted in one of the four chambers mocked up as a typical office environment with desks and computer workstations. Four participants and one researcher were present during test periods, shown in Figure 1 (a) and (b). An experimental timeline, post-occupancy by test subjects, is shown in Figure 1 (c). The test chamber was served by a single-pass, mixing ventilation forced air heating, ventilation, and air-conditioning (HVAC) system that includes supply air cleaning, with MERV8 and pleated activated carbon (International Filtration, Carbon Pleated Interfold). Supply air is drawn into the chamber HVAC system from the surrounding indoor space, which is a laboratory environment. A ceiling fan with transparent blades (diameter $=1.47 \mathrm{~m}$, Artemis Minka-Aire F803L-TL model, 

shown in Table S1 and Table S2 of the Supporting Information.
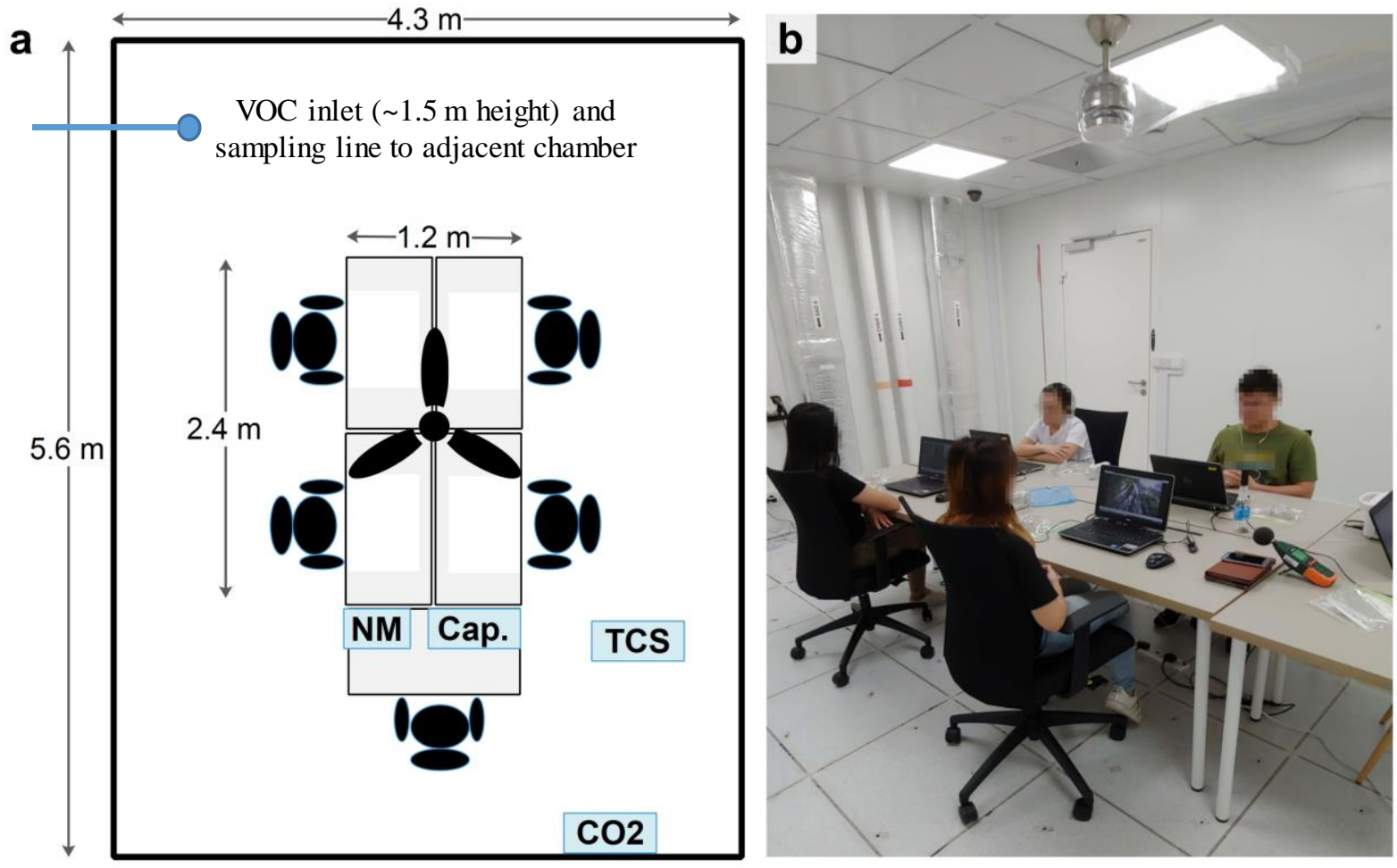

C

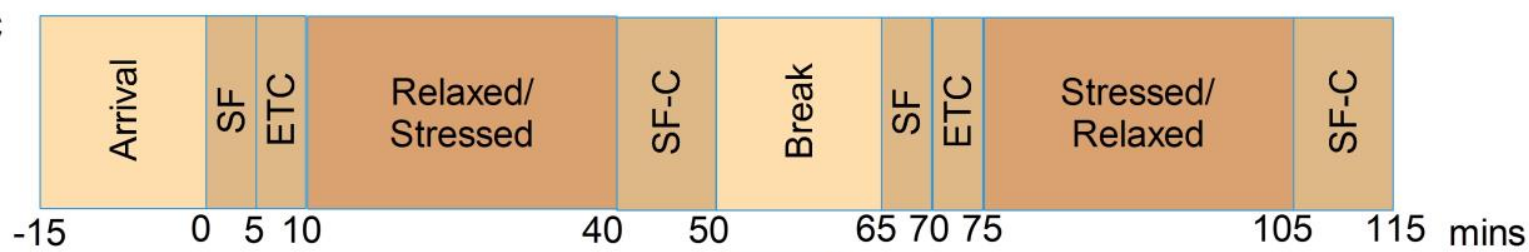

Figure 1. Overview of experimental set-up (a) and (b) and time-line of experiments postoccupancy (c). SF = subjective feedback, ETC = eye tracker calibration, $\mathrm{SF}-\mathrm{C}=$ subjective feedback \& capnometer, TCS $=$ thermal comfort monitoring station, $\mathrm{CO}_{2}=$ Wall mounted $\mathrm{CO}_{2}$ sensor, $\mathrm{NM}=$ noise meter, $\mathrm{Cap} .=$ capnometer.

\subsection{Measurement of participant stress levels}

Our test protocol recruited participants to engage in an experiment where human chemical emissions were measured while engaged in two activities: a "relaxed" activity where the participants viewed a 30-minute nature documentary (two clips of nature documentaries, 15 min each, the first a "tour" of redwood forests and the second a hummingbird documentary 
narrated by Sir David Attenborough) and a "stressed" activity where the participants engaged in a 30-min series of cognitive tasks. We selected five cognitive tasks covering the executive functions: working memory, inhibition, and cognitive flexibility/task switching. ${ }^{39}$ The tasks were administered through the PEBL platform. ${ }^{40}$ Prior studies suggest that audiovisual stimuli can alter stress state, observable by biomarkers like cortisol and salivary alpha amylase, ${ }^{41,42}$ with viewing of stressful or soothing videos of $\sim 30$ min in length. ${ }^{42,43}$ We include further details of cognitive tests used in the Supporting Information. In total, we tested sixteen participants in groups of four, with the order of exposure to the relaxed and stressed activities alternating over the four days of testing to achieve a counterbalanced design. Participant characteristics are shown in Table S3 of Supporting Information. The test protocol was approved by the University of California Berkeley Ethics Committee (Protocol \#IRB2019-07-12403). Participants were briefed on the study in a one-hour familiarization session conducted for each participant prior to the experiments.

We measured objective and subjective indicators of occupant stress and perceptions of the test-chamber's indoor environment. Participants arrived 15 minutes prior to the start of the session and were outfitted with a heart rate sensor (Polar H10, Polar, Finland). During the session, each participant sat at a workstation with a laptop. Each laptop had an eye tracker installed (Tobii Pro Nano, Tobii Pro, Sweden) to measure pupil dilation. Upon completion of the relaxed and stressed activities, we measured salivary alpha amylase (Cocoro Meter, Nipro Corporation, Japan) and end tidal $\mathrm{CO}_{2}\left(\mathrm{etCO}_{2}\right)$ level $\left(\mathrm{CO}_{2}\right.$ monitor, OLG-3800, Nihon Kohden, Japan). We also collected subjective feedback concerning indoor environmental quality and mental effort; further details are provided in the Supporting Information. After the 
141 session was complete, we measured participants' weight and body composition using a

142 bioelectrical impedance based monitor (RD-545IM, Tanita, Japan).

143

144

145

146

147

148

149

150

151

152

153

154

155

156

157

158

159

160

161

162

163

164

\subsection{Human chemical emission measurement}

Human chemical emissions evaluated in this study include carbon dioxide and isoprene. Human chemical emissions were measured during a 30-min baseline period, occurring prior to the arrival of occupants, and during the two 30-min periods during occupancy, coinciding with relaxed or stressed activity (Figure 1 (c), baseline not shown). In 1-min intervals, measurements of $\mathrm{CO}_{2}$ were made in the chamber supply air and in bulk air in the test chamber (GMW84, Vaisala, Finland, Range 0-2000 ppm, uncertainty: larger of 30 ppm and 3\%). Time-integrated measurements of volatile organic compounds were made during baseline and occupied periods via sampling with sorbent tubes packed with $100 \mathrm{mg}$ of Tenax TA and $180 \mathrm{mg}$ of Carbograph 1 , based on a method developed previously, ${ }^{44}$ modified for target compounds, sampling times, and sampling flowrate $(\sim 130 \mathrm{~mL} / \mathrm{min}$, total sample volume $=4 \mathrm{~L})$. One portable sampling pump $(\mathrm{PCXR} 4, \mathrm{SKC}, \mathrm{USA})$ drew sample air through perfluoroalkoxy (PFA) tubing connected to the room supply air duct and another sampling pump did the same from the bulk room air. Both sampling pumps had $\sim 2 \mathrm{~m}$ of 0.635 " OD tubing placed upstream of the sorbent cartridge. Bulk room air samples were made in duplicate using a flow splitter. Sorbent cartridges were analyzed with thermal desorption - gas chromatography - mass spectrometry (TD-GC-MS, TurboMatrix 650, PerkinElmer, USA connected to a gas chromatograph, Model 7890 A, Agilent Technologies, USA). We quantified VOCs by generating calibration curves from a calibration mixture that included isoprene and acetone. We do not report acetone levels or emission rates due to uncertainty in quantification, apparent in high variability across duplicate samples: the mean relative percent difference (RPD) across all duplicate isoprene concentrations measured was $10.9 \%$ while the 
RPD for acetone duplicates was $66.8 \%$. Since acetone is emitted by humans with high source strength, we suspect the method is not suitable for robust acetone quantification at the levels present in the chamber. Further details of the sampling and analysis method are provided in the Supporting Information and in Figure S2. To return chamber bioeffluent levels to nearbackground levels, during the break between the two activities the room was flushed with a fan (flowrate $=62 \mathrm{~m}^{3} / \mathrm{min}, \mathrm{IBF} 300$, Aman, India) providing $\sim 10$ turnovers of air during the 15-min break.

Chamber temperature and relative humidity were measured continuously in 1-min intervals (ThermCondSys 5500, Sensor, Poland) and ozone levels were monitored (Model 205, 2BTech, USA) once per day, prior to the arrival of occupants, to ensure near-zero ozone levels; ozone was consistently $<2 \mathrm{ppb}$, the stated uncertainty of the instrument. Light levels at each workstation ranged between 931 and 957 lux, which is within normal ranges of typical office work environments. The chamber windows were blacked out to ensure consistent lighting levels and avoid variations due to outside lights. We determined the air-exchange rate in the chamber from measurements of air flow rates in the supply duct as well as tracer decay tests conducted following the conclusion of the experiments for the day (excepting day 1) (see Table S1). The chamber is well-mixed, confirmed via measurements of temperature and airflow gradients, as well as high $r^{2}(>0.99)$ when applying a well-mixed model to $\mathrm{CO}_{2}$ tracer decay periods. The sorbent tubes and sampling pumps were located in a chamber adjacent to the experimental test chamber, and were not visible or audible to participants (instruments were in Chamber III, participants in Chamber IV, Figure S1 of Supporting Information).

\subsection{Data analysis \\ 3.4.1 Calculation of $\mathrm{CO}_{2}$ emission rates}



baseline and 30-minute periods during which participants were engaged in either relaxed or stressed activity. We used a non-linear curve-fit applied to the analytical solution of a massbalance equation that included air-exchange and $\mathrm{CO}_{2}$ emissions from occupants, shown in 192 equation 1:

$$
C_{C O 2, t}=\left(C_{C O 2, o}+\frac{S_{C O 2}}{\lambda V}\right)\left(1-e^{-\lambda t}\right)+C_{C O 2, t=0} e^{-\lambda t}
$$

193 where, $C_{C O 2, t}$ is the concentration of $\mathrm{CO}_{2}$ in the chamber at time $t$ within the 30-min period of a 194 "relaxed" or "stressed" activity $\left(\mathrm{g} \mathrm{m}^{-3}\right), C_{C O 2, o}$ is the time-averaged concentration in supply air 195 over the 30-min period $\left(\mathrm{g} \mathrm{m}^{-3}\right), S_{\mathrm{CO} 2}$ is the chamber $\mathrm{CO}_{2}$ source strength due to occupancy (g $\left.196 \mathrm{~h}^{-1}\right), \lambda$ is the air-exchange rate due to ventilation of the chamber $\left(\mathrm{h}^{-1}\right), V$ is the volume of the 197 chamber $\left(\mathrm{m}^{3}\right), t$ is time since the start of the 30 -min test period (h), and $C_{C O 2, t=0}$ is the $\mathrm{CO}_{2}$ 198 level in the chamber at the start of the 30-min test period $\left(\mathrm{g} \mathrm{m}^{-3}\right)$.

A best-fit value of $S_{\mathrm{CO} 2}$ over the 30 -min period was determined using the "fit" function 200 in MATLAB R2019a, with the fit type specified as eq. 1. The chamber was occupied by five 201 persons during all occupied test conditions; we report $\mathrm{CO}_{2}$ source strengths on a per-person 202 basis by dividing the best-fit $S_{\mathrm{CO} 2}$ from eq. 1 by five. Note that one of the occupants was 203 always the same researcher, present in the chamber as per IRB protocol requirement. He 204 maintained a similar diet, exercise level, and sleep schedule during the week of testing, and 205 endeavored to maintain similar during-test activity level across all tests.

\subsubsection{Calculation of isoprene emission rates}

207 Isoprene was measured over three 30-min periods: baseline (unoccupied), stressed and 208 relaxed activity (both occupied by five persons). Measured isoprene levels are time-integrated 209 over these 30-min periods. In the case of the baseline period, the chamber was previously 
unoccupied and operated at constant air-exchange for $\sim 18$ hours, and we assume the chamber

211 has reached steady-state. For unoccupied conditions we calculate the isoprene emission rate

212 according to equation 2:

$$
S_{\text {isoprene,baseline }}=\lambda\left(C_{i, \text { chamber }}-C_{i, o}\right) \mathrm{V}
$$

213 where $S_{\text {isoprene,baseline }}$ is the source or sink strength of isoprene $\left(\mu \mathrm{g} \mathrm{h}^{-1}\right)$ under baseline

214 conditions, $\lambda$ is the air-exchange rate due to ventilation of the chamber $\left(\mathrm{h}^{-1}\right), C_{i}$ is the

215 concentration of isoprene in chamber air $\left(\mu \mathrm{g} \mathrm{m}^{-3}\right), C_{i, o}$ is the concentration of isoprene in

216 supply air $\left(\mu \mathrm{g} \mathrm{m}^{-3}\right)$ and $V$ is the volume of the chamber $\left(\mathrm{m}^{3}\right)$. Measurements of isoprene were

217 corrected for the field blank, converted from mass to concentration using the sample volume

218 for the specific sampling event.

219 For periods of occupancy, we developed a method for estimating the isoprene source

220 strength using a mass-balance model as in equation 1, written for isoprene. Because our

221 measurement of isoprene was time-integrated, we integrated the mass-balance model over the

222 30-min period of relaxed or stressed condition, in 1-min time-step, for either relaxed or

223 stressed activity period to determine the area under the concentration-time curve. Note that the

224 relaxed or stressed condition began 10 min following occupancy (Figure 1c). This area

225 calculation was established as the objective function, and the source strength of isoprene over

226 the 30-min period was varied until the modeled area is equal to that of the concentration-time

227 area known from the time-integrated measurement, as shown in equation 3:

$$
\begin{gathered}
\sum_{t=10}^{40 \mathrm{~min}} C_{i, \text { model, } t} \times \Delta t_{\text {model }}=C_{i, \text { measured }} \times \Delta t_{\text {measured }} \\
228 \quad \text { where } C_{i, \text { model }, t} \text { is the modeled chamber concentration of isoprene, calculated in 1-min }
\end{gathered}
$$

229 intervals using a mass-balance as shown in equation S1 of the Supporting Information $\left(\mu \mathrm{g} \mathrm{m}^{-}\right.$

$230{ }^{3}$ ), $\Delta t_{\text {model }}$ is the time resolution of the discrete integration of the model concentration-time 
231 curve (1 min), $C_{i, m e a s u r e d}$ is the time-integrated measurement of isoprene in the chamber,

232 taken as the average of duplicate measurements of isoprene and corrected for field blank $(\mu \mathrm{g}$

$\left.233 \mathrm{~m}^{-3}\right)$, and $\Delta t_{\text {measured }}$ is the time duration of the measured isoprene level in the chamber (30-

$234 \mathrm{~min})$.

235 A detailed example of the method employed for determining isoprene emission rates is

236 provided in the supporting information in Table S4, with a graphical presentation Figure S3,

237 present in the Supporting Information.

$238 \quad 3.4 .3$ Statistical tests

239 As shown in Table S1 of the Supporting Information, we conducted tests over four

240 days where $\mathrm{CO}_{2}$ and isoprene source or sink strengths were measured for baseline, relaxed,

241 and stressed activity, with order of relaxed and stressed activity alternating each day. To

242 determine if the test conditions resulted in statistically significant changes in $\mathrm{CO}_{2}$ or isoprene

243 source or sink strength in the chamber, we used analysis of variance (ANOVA) across three

244 groups (baseline, relaxed, stressed), similar to a method described previously. ${ }^{45}$ Briefly, if the

245 three-group comparison resulted in $\alpha<0.05$, we then made direct comparisons across groups

246 (i.e., baseline vs. relaxed, baseline vs. stressed, and relaxed vs. stressed) using Tukey’s honest

247 significant difference test (Tukey test). We included the baseline (unoccupied) conditions in

248 ANOVA to evaluate whether source or sink strengths during occupied conditions were

249 significantly different from unoccupied conditions. All statistical analyses were performed

250 using MATLAB 2019a.

251 Measurements of biomarkers (HRV, SAA, pupil dilation, and end tidal $\mathrm{CO}_{2}$ ) of stress

252 were analyzed using either parametric (t-tests) or non-parametric tests (Wilcoxon rank sum

253 tests) depending on if the data was normally distributed or not. Significance level was taken at 
2540.05 except for the HRV parameters, where, due to the large number of comparisons,

255 Benjamini-Hochberg procedure (false discovery rate taken as 10\%) was used. All

256 comparisons were paired and two tailed.

257 Data and analysis scripts/functions are publicly available and can be downloaded from

258 the Dryad repository: https://doi.org/10.5061/dryad.gb5mkkwmk.

\section{4. Results and Discussion}

2604.1 Objective and subjective indicators of occupant stress

261 Objective measured stress biomarkers shown in Figure 2 indicate that the cognitive

262 tests (stressed activity) induced a state of occupant stress compared to watching nature

263 documentaries (relaxed activity). While most of the physiological parameters examined

264 should increase with stress levels, the inverse is true for some parameters, specifically the

265 Mean RR interval (the interval between heart beats) and fraction of power of heart beats in the

266 lower frequency bands. To create a simpler visual presentation, we present these parameters

267 multiplied by -1 for plots. This ensures that all the plotted parameters are low when stress is

268 low and are high when stress is high.

269 

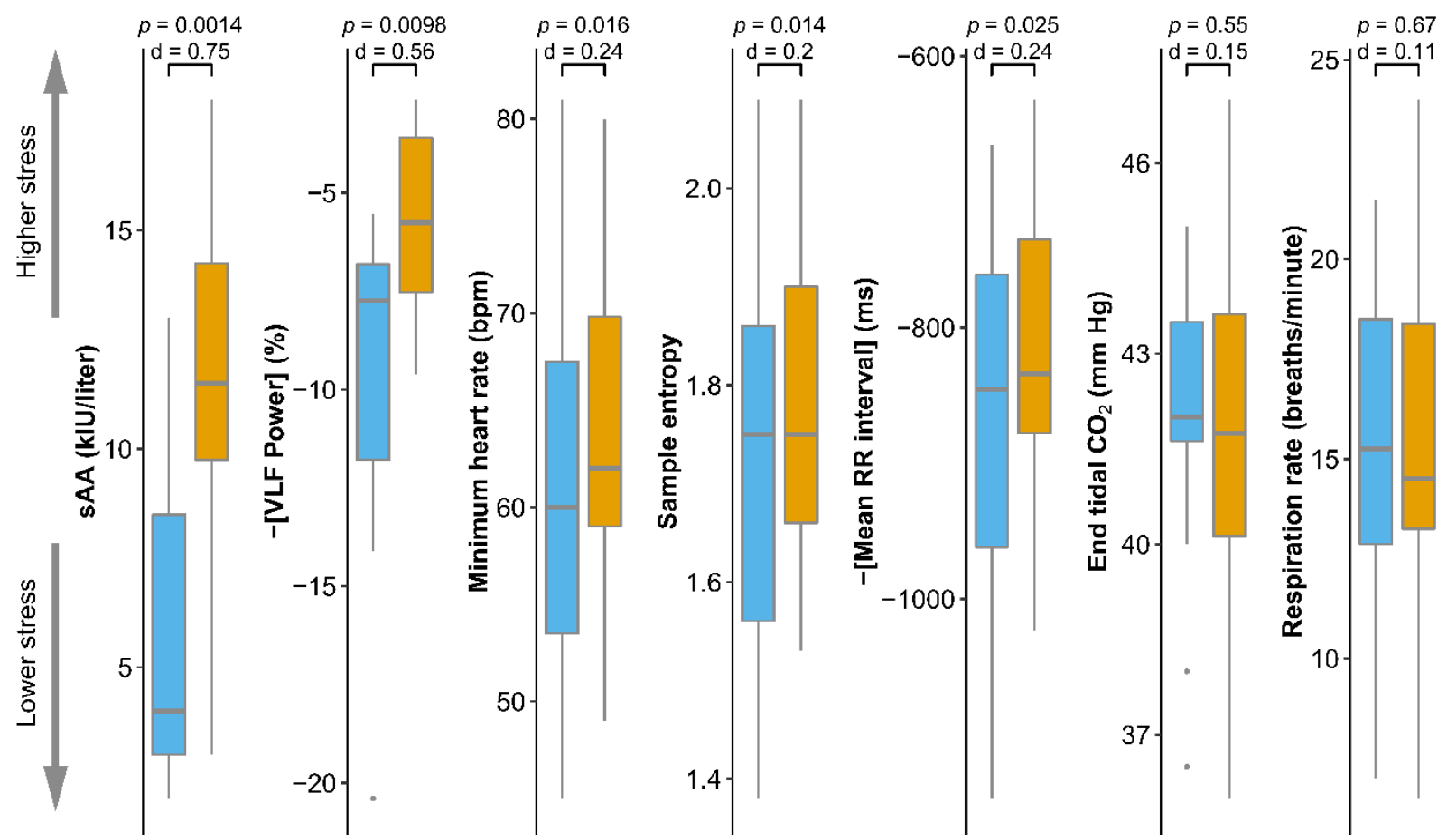

Figure 2. End tidal $\mathrm{CO}_{2}$, salivary alpha amylase (sAA), respiration rate and summary of key indicators of heart rate variability data: fraction of power in the very low frequency (VLF) band, minimum heart rate, sample entropy and mean of the RR interval (interval between heart beats) measured in the relaxed and stressed activity.

Salivary alpha amylase levels are elevated during stressed activity compared to relaxed activity ( $p$-value $=0.0014, \mathrm{~d}=0.75$, a medium to large size effect). Salivary alpha amylase levels are known to follow a diurnal course, and prior studies recommend controlling for time of day; ${ }^{46}$ our design had occupants entering the chamber at 14:00 (Table S1) each day and the relaxed/stressed activities occurred over a period of $\sim 120 \mathrm{~min}$. Nevertheless, the study was counterbalanced, which will address the potential for confounding due to diurnal changes in salivary alpha amylase that may have occurred over this 120 -min period, though we expect this effect to be small based on the diurnal profiles presented by Nater et al. ${ }^{46}$. We analyzed 51 parameters of heart rate variability; as noted from previous studies, ${ }^{47}$ not all parameters are expected to yield a significant difference when stress is imposed on people. Note that one participant's data was lost on day 3; subsequent analysis used data from fifteen participants. In 
286 Figure 2 are four metrics showing differences due to external stress, evident due to

287 statistically significant differences from the relaxed to stressed activity in the nonparametric

288 statistical tests and Cohen's D. The VLF power is the percentage of power in the Very Low

289 Frequency band $(0.003 \leq \mathrm{f}<0.04 \mathrm{~Hz})$ of the frequency domain transformation (Fast Fourier

290 Transform) of the time domain RR (interval between heart beats) interval data ( $\mathrm{p}=0.0098, \mathrm{~d}=$

291 0.56, a medium size effect). The degree of heart rate variability (HRV) in terms of VLF power

292 is expected to reduce as activity intensity increases, ${ }^{48}$ an effect observed in our data. The

293 minimum heart rate was lower during relaxed activity $(\mathrm{p}=0.016, \mathrm{~d}=0.24$, a small size

294 effect). Sample entropy, which indicates randomness of the data (lack of regularity in heart

295 rate), was higher for the stressed activity $(\mathrm{p}=0.014, \mathrm{~d}=0.2$, a small size effect). Finally, the

296 mean RR was significantly higher during the relaxed activity, indicating greater heart rate

297 variability and hence less stress $(p=0.025, d=0.2$, a small size effect). Results of subjective

298 feedback assessments (sleepiness, thermal preference) and pupil dilation data are provided in

299 the Supporting Information. Mean and median pupil size were significantly larger during

300 stressed than relaxed activity, shown in Figure S4 of the Supporting Information, another

301 objective indicator associated with stress. ${ }^{49}$ Collectively, these objective indicators

302 demonstrate that cognitive testing vs. nature documentary was effective in causing a relative

303 increase in participants' stress.

304 The end-tidal $\mathrm{CO}_{2}\left(\mathrm{etCO}_{2}\right)$ and respiration rate data did not show significant difference

305 between relaxed and stressed activity periods $(\mathrm{p}>0.7)$, determined using a t-test since

306 distributions were normal. This finding was somewhat unexpected given that emission rates of

$307 \mathrm{CO}_{2}$ calculated from chamber air during occupied periods (Section 4.3) are elevated during

308 stressed compared to relaxed activity. Importantly, etCO $\mathrm{C}_{2}$ was measured post-testing, over a 
309 period of $\sim 10$ minutes following completion of relaxed or stressed activity while bioeffluent

$310 \mathrm{CO}_{2}$ emission rates are calculated with data collected during the 30-min of relaxed or stressed

311 activity. No significant changes in etCO $\mathrm{CO}_{2}$ implies body $\mathrm{CO}_{2}$ levels were similar at the end of

312 the two periods. As respiration rates are also similar, in conjunction with etCO $\mathrm{O}_{2}$ values, this

313 indicates that the body is able to effectively ventilate the additional $\mathrm{CO}_{2}$ being generated

314 during the test period, possibly through breathing in greater volumes of air.

3154.2 Human emissions of $\mathrm{CO}_{2}$ and isoprene

316 Representative experimental results from one of the four days of controlled testing of

317 human chemical emissions are shown in Figure 3. An abbreviated experimental protocol is

318 shown: measurement of levels of chemical emissions occurred during a baseline (unoccupied)

319 period and two subsequent occupied periods. Also shown in Figure 3 is example TD-GC-MS

320 data for isoprene, which is time-integrated. The $\mathrm{x}$-direction bars on the plot indicate the period

321 of time over which the sample was taken while the y-direction error bars show the range

322 across duplicate samples taken in chamber air. 


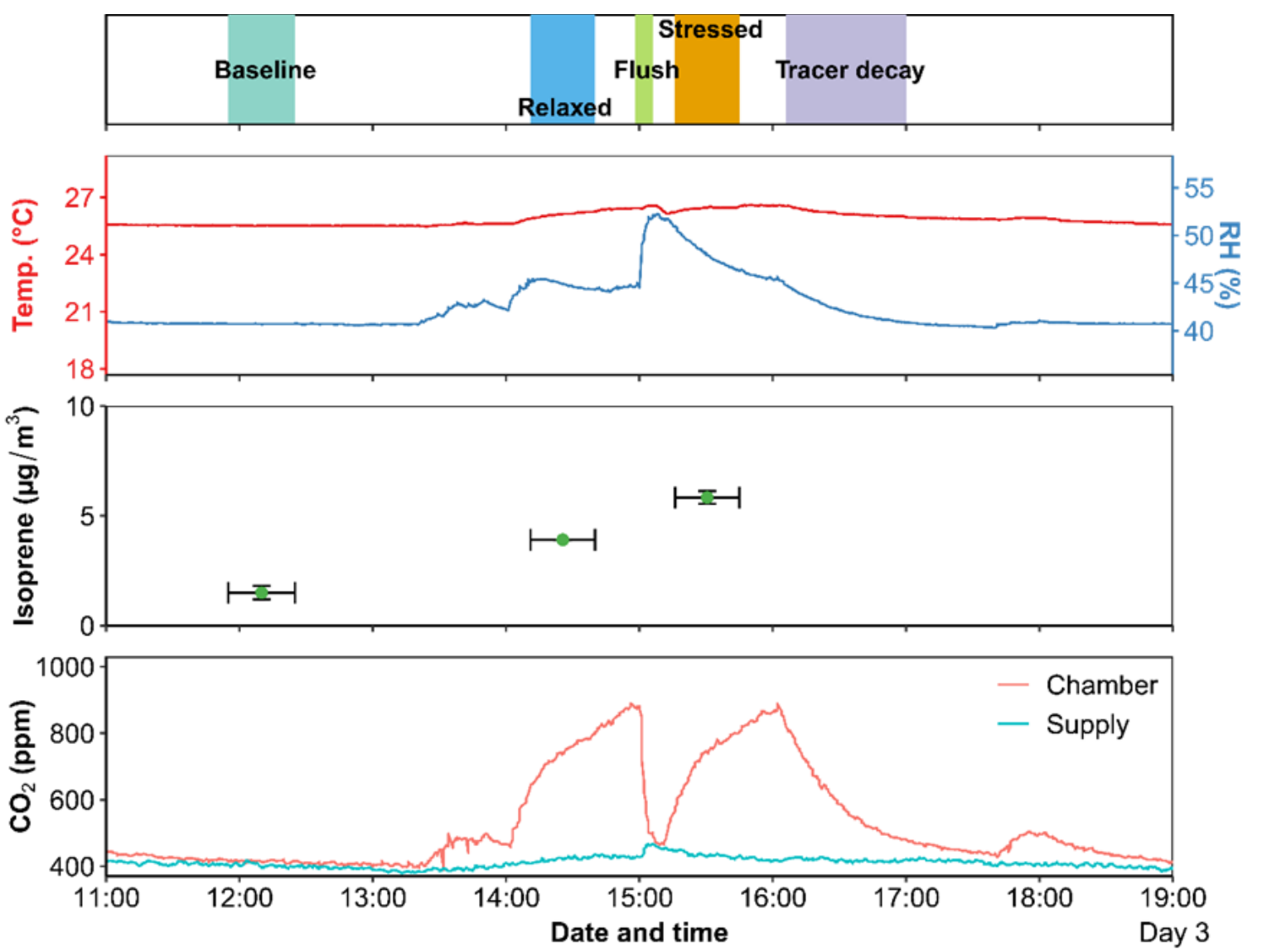

Figure 3. Representative experimental results for chamber dry-bulb air temperature, relative humidity, isoprene levels during baseline and occupied periods and time-series measurements of carbon dioxide.

The effect of occupancy on $\mathrm{CO}_{2}$ levels and the flushing of the chamber in between the two occupied periods is apparent in Figure 3, the flushing due to a temporarily placed fan that evacuated air from the chamber, replacing with ambient laboratory air and reducing to nearbackground levels of $\mathrm{CO}_{2}$ prior to the initiation of the second test activity. The test chamber $\mathrm{CO}_{2}$ level was monitored and observable in real-time, and was used to ensure the second test activity of each day occurred with initial $\mathrm{CO}_{2}$ conditions similar to that of the first test. The short duration for flushing the chamber between occupied activities is one reason for limiting analysis of chemical emissions to $\mathrm{CO}_{2}$ and isoprene, both compounds that are predominantly present in the gas-phase. There existed no aqueous water present in the chamber and surfaces were impermeable, hard surfaces. $\mathrm{CO}_{2}$ sorption to surfaces indoors is typically taken as 
337 negligible. Isoprene is considered a very volatile organic compound, or VVOC, with vapor

338 pressure $7.2 \times 10^{4} \mathrm{~Pa} .{ }^{50}$ A recently published model of partitioning of organics indoors shows

339 isoprene is expected to be predominantly present in the gas-phase across a range of indoor

340 surface reservoir conditions, though experimentally determined response times indicate

341 dynamic partitioning does occur. ${ }^{51} \mathrm{We}$ expect that error introduced for isoprene from surface

342 partitioning is likely small, based on calculations of emission rates into the chamber under

343 unoccupied conditions vs. occupied conditions (13 $\mu \mathrm{g} / \mathrm{h}$ vs. $675 \mu \mathrm{g} / \mathrm{h})$. Furthermore,

344 uncertainty due to the impact of isoprene surface interactions on comparisons of emission

345 magnitudes across stressed and relaxed activities is addressed, at least partially, in the

346 counterbalanced design.

We calculated source strengths during baseline, relaxed and stressed activities for $\mathrm{CO}_{2}$

348 and isoprene. Results of the source strength calculation are shown in Table 1 for isoprene and

$349 \mathrm{CO}_{2}$, which met the three-group comparison ANOVA criteria of $\alpha<0.05$.

350 Table 1. Chamber conditions and magnitudes of carbon dioxide and isoprene emissions

351 across baseline, relaxed, and stressed activities.

\begin{tabular}{|c|c|c|c|c|c|c|c|c|c|c|c|}
\hline Day & & Temp & $\mathbf{R H}$ & $\mathrm{O}_{3}$ & $\begin{array}{c}\mathrm{CO}_{2} \\
\text { inlet* }\end{array}$ & $\begin{array}{c}\mathrm{CO}_{2} \\
\text { chamber* }\end{array}$ & $\begin{array}{c}\text { Isoprene } \\
\text { inlet }^{\%}\end{array}$ & \multicolumn{2}{|c|}{$\begin{array}{l}\text { Isoprene } \\
\text { chamber }^{2}\end{array}$} & $\begin{array}{c}\mathrm{CO}_{2} \\
\text { emission } \\
\text { rate }\end{array}$ & $\begin{array}{c}\text { Isoprene } \\
\text { emission } \\
\text { rate }\end{array}$ \\
\hline & & ${ }^{\circ} \mathrm{C}$ & $\%$ & $\mathrm{ppb}$ & ppm & $\mathrm{ppm}$ & $\mu \mathrm{g} / \mathrm{m}^{3}$ & \multicolumn{2}{|c|}{$\mu \mathrm{g} / \mathrm{m}^{3}$} & $g / h / p$ & $\mu g / h / p$ \\
\hline & & & & & & & & rep1 & rep2 & & \\
\hline \multirow{3}{*}{1} & Baseline $^{\#}$ & 26.9 & 38 & $<2$ & 415 & 420 & 2.1 & 1.5 & 2.1 & 0.32 & 8.03 \\
\hline & Relaxed & 26.9 & 43 & & 441 & 784 & 2.4 & 4.9 & 5.4 & 27.8 & 144.2 \\
\hline & Stressed & 27.1 & 48 & & 467 & 768 & 2.8 & 5.6 & 5.7 & 30.5 & 154.9 \\
\hline \multirow{3}{*}{2} & Baseline $^{\#}$ & 25.6 & 41 & $<2$ & 411 & 435 & 1.8 & 1.9 & 1.9 & 0.65 & -2.72 \\
\hline & Stressed & 26.2 & 46 & & 406 & 774 & 1.9 & 4.8 & 3.9 & 32.3 & 119.3 \\
\hline & Relaxed & 26.5 & 47 & & 441 & 722 & 1.9 & 4.5 & 4.0 & 27.7 & 115.6 \\
\hline \multirow{3}{*}{3} & Baseline $^{\#}$ & 25.5 & 41 & $<2$ & 403 & 415 & 1.6 & 1.7 & 1.4 & 0.58 & 2.84 \\
\hline & Relaxed & 26.1 & 45 & & 421 & 733 & 2.0 & 3.9 & 3.9 & 25.4 & 102.5 \\
\hline & Stressed & 26.4 & 48 & & 435 & 721 & 2.6 & 5.7 & 6.0 & 28.9 & 176.4 \\
\hline \multirow{3}{*}{4} & Baseline $^{\#}$ & 25.5 & 40 & $<2$ & 403 & 418 & 2.2 & 2.5 & 2.2 & 0.69 & 2.75 \\
\hline & Stressed & 26.2 & 45 & & 420 & 780 & 2.6 & 5.8 & 6.1 & 29.7 & 165.9 \\
\hline & Relaxed & 26.5 & 47 & & 430 & 712 & 2.1 & 4.1 & 4.5 & 27.3 & 100.0 \\
\hline
\end{tabular}


* $\mathrm{CO}_{2}$ levels were measured in 1-min interval in the inlet and room, averages over the 30-min test periods are reported here for brevity. See Figure $\mathrm{S} 5$ for full time-series analysis of $\mathrm{CO}_{2}$ data. \# baseline $\mathrm{CO}_{2}$ and isoprene emission rates are presented on a per-person normalized basis, though no humans were present in the chamber during baseline conditions, to enable comparisons to the occupied (relaxed and stressed activities). All per-person normalizations were made by dividing the total emission rate into the chamber by five, four participants plus the experimenter. $\%$ isoprene levels were corrected for field blanks in calculations of isoprene source strength (field blanks were $0.27,0.27,0.27$, and $0.45 \mu \mathrm{g} / \mathrm{m}^{3}$ on days, $1,2,3$ and 4 , respectively)

\section{Emission rates calculated from mass-balance models during accumulation of $\mathrm{CO}_{2}$ in}

the test chamber $(28.7 \mathrm{~g} / \mathrm{h} / \mathrm{p}$ averaged across all participants and conditions $)$ are in agreement with prior estimates of per-person $\mathrm{CO}_{2}$ generation rates. Values reported here are consistent with estimates for adults in residences and bedrooms, and slightly lower than values typically assumed for office environments. ${ }^{1,18}$ Per-person $\mathrm{CO}_{2}$ emission rates reported here are similar to those reported by Stönner et al. ${ }^{28}$ for adults $(30 \mathrm{~g} / \mathrm{h} / \mathrm{p})$ and $\mathrm{Wang}^{35}$ for students $(27 \mathrm{~g} / \mathrm{h} / \mathrm{p}$ during lecture, $38 \mathrm{~g} / \mathrm{h} / \mathrm{p}$ during exam). Our results are somewhat higher than the average reported by Tang et al. ${ }^{2}$ in an occupied college classroom $(21 \mathrm{~g} / \mathrm{h} / \mathrm{p})$. Our per-person emission rate for isoprene is $135 \mu \mathrm{g} / \mathrm{h} / \mathrm{p}$ across all occupied conditions $(132 \mu \mathrm{g} / \mathrm{h} / \mathrm{p}$ if the aforementioned baseline emission is accounted for), are in general agreement with prior studies. Three independent prior studies reporting per-person isoprene emission rates for adults are 105,162 , and $166 \mu \mathrm{g} / \mathrm{h} / \mathrm{p} .^{2,27,28}$ Our estimate for per-person isoprene emission rate is near the average of these prior studies.

\subsection{Impact of cognitive tasks on isoprene and $\mathrm{CO}_{2}$ emission rate}

Full results across the four days of repeated testing are shown in Figure 4 for carbon dioxide and isoprene. Per-person $\mathrm{CO}_{2}$ emission rates were greater for stressed than relaxed conditions $(30.3 \pm 2.1$ vs. $27.0 \pm 1.7 \mathrm{~g} / \mathrm{h} / \mathrm{p}, p=0.0044$, mean \pm standard deviation $)$ and 
381 isoprene emission rates were also elevated under stressed vs. relaxed conditions (154 \pm 25

$382 \mu \mathrm{g} / \mathrm{h} / \mathrm{p}$ vs. $116 \pm 20 \mu \mathrm{g} / \mathrm{h} / \mathrm{p}, p=0.041)$
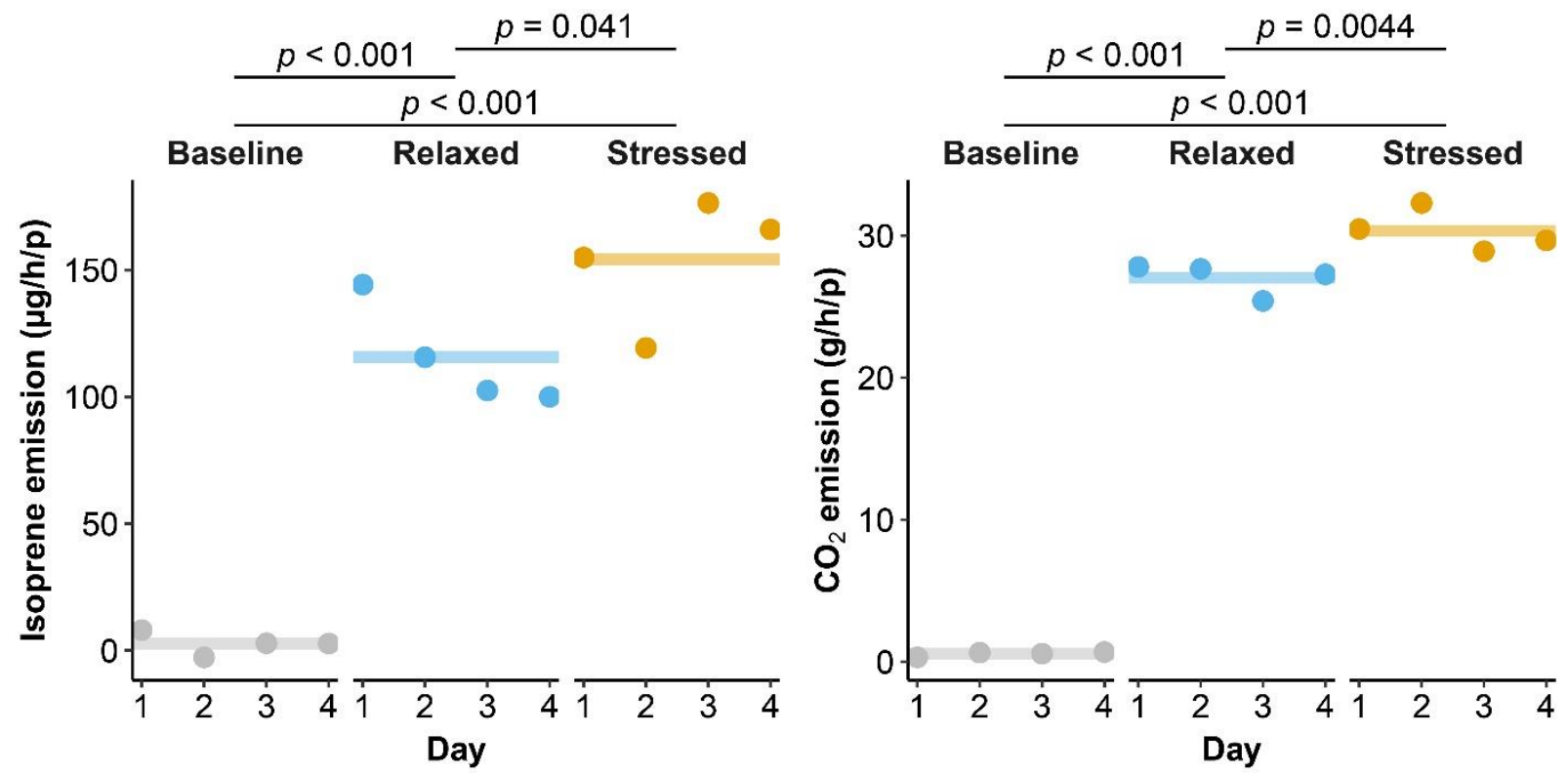

Figure 4. Per-person emission rates of carbon dioxide and isoprene. The horizontal bar is the average across all days of testing, each dot shows the measurement for a particular day. Values of emission rates shown for occupied condition emissions (i.e., relaxed and stressed activities) do not subtract unoccupied (baseline) estimates of emissions.

Shown in Figure 4 is that carbon dioxide emission rates are consistently higher (12\%)

during stressed activity than relaxed activity for all repeated trials of the study; regressions used to estimate emission rates from dynamic $\mathrm{CO}_{2}$ data each day of testing are shown in Figure S5 of the Supporting Information. Because the study was counterbalanced, consistently elevated $\mathrm{CO}_{2}$ emissions imply that it is the stressed activity that caused the elevated $\mathrm{CO}_{2}$ emission, rather than the order of tests conducted. Inspection of isoprene emission rates also reveals elevated emission rate under stressed activity; isoprene emissions were $33 \%$ higher than the relaxed activity. The finding of greater relative increases in isoprene vs. $\mathrm{CO}_{2}$ emission rate is interesting given that demand-controlled ventilation systems are typically based on $\mathrm{CO}_{2}$ levels; results here indicate isoprene may be a useful additional parameter for designing building ventilation systems with sensitivity to cognitive tasks. 
As presented in Section 4.1, we measured objective indicators of occupant stress,

401

402

403

404

405

406

407

408

409

410

411

412

413

414

415

416

417

418

including biomarkers of salivary alpha amylase and metrics associated with participant's heart

rate variability and pupil dilation. We also measured chamber temperature and relative

humidity conditions. To explore the utility of these objective biomarkers and environmental

conditions as possible predictors of the observed elevation in bioeffluent emission rates, we

performed regression to observe relationships to variance and change in $\mathrm{CO}_{2}$ and isoprene

emission rates. Full results of this analysis are shown in Figure S6 of Supporting Information

for biomarkers observed to be statistically significantly different between stressed and relaxed activity and temperature and RH. Note that since bioeffluent emission rates are the average per-person emission for individuals present in the chamber, we regress these emission rates against biomarkers averaged for those same individuals. The sAA data here includes that of the experimenter who was inside the chamber, was not subject to the same research protocol, but nevertheless contributed to $\mathrm{CO}_{2}$ and isoprene emissions. However, since the experimenter did not wear an HRV monitor, the HRV related markers do not have his information.

We found that of biomarkers measured, only subject salivary alpha amylase (Figure 5) is significantly related to isoprene emission rates $(p$-value $=0.02)$, and that a substantial portion of the variance in observed isoprene emission rates is explained $\left(R^{2}=0.60\right)$. This result is interesting considering a prior study identifies isoprene as a marker associated with emotional stress arising from moviegoers' interaction with audiovisual stimuli during films. ${ }^{36}$ Stönner et al. ${ }^{37}$ suggest that isoprene emission and variability in isoprene level could be useful as an objective indicator for recommending age-appropriateness of different films. Isoprene is thought to be generated during cholesterolgenesis ${ }^{52}$ and some studies suggest measurement of breath isoprene levels may be useful as a non-invasive method for assessing blood cholesterol 
423 levels. ${ }^{53}$ Williams et al. ${ }^{36}$ suggest isoprene production is related to cortisol production via the 424 cholesterol production pathway; this link appears based on their empirical findings, as no 425 biological pathway is mentioned. If isoprene and cortisol production are related, this may 426 explain the observed correlation here between isoprene emission and salivary alpha amylase, 427 since studies show responses of both salivary alpha amylase and cortisol follow stressful 428 events with salivary alpha amylase levels responding more rapidly than cortisol levels. ${ }^{54}$ 429 However, it should be cautioned that linking the production of an endogenously generated 430 compound like isoprene to external stimuli is challenging, as per-person differences in 431 isoprene production as measured by human breath may result from interactions between the 432 metabolic pathways with the circulatory (heart rate) and pulmonary systems (breathing rate). ${ }^{53}$ 

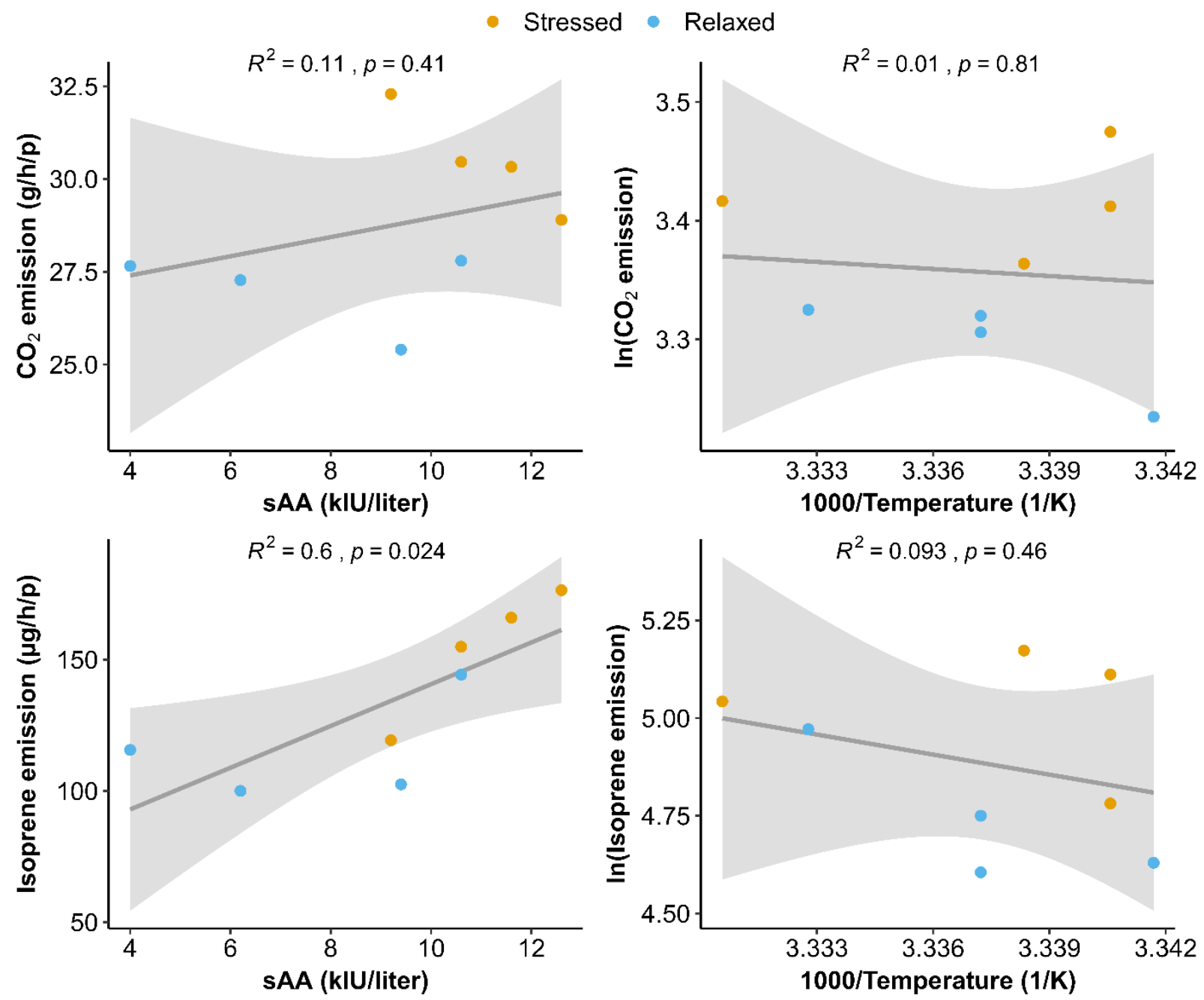

Figure 5. Regression of isoprene and $\mathrm{CO}_{2}$ emission rates vs. salivary alpha amylase and chamber air temperature. Shown above each plot are the r-squared and $p$-value associated with the linear regression slope. Curves around each linear regression are the $95 \%$ confidence interval. Note that the target chamber temperature was constant at $26{ }^{\circ} \mathrm{C}$; the above plot investigates whether incidental variation in temperature is related to observed changes in in emission rates of isoprene and $\mathrm{CO}_{2}$.

Changes in salivary alpha amylase were not significantly associated with changes in per-person $\mathrm{CO}_{2}$ production rates. It is possible that $\mathrm{CO}_{2}$ production was elevated due to temporary increase in metabolism during the stressed activity, but was not correlated with endogenous production of salivary alpha amylase. Other biomarkers measured associated with heart rate did not appear to have statistically significant correlation with changes in isoprene or $\mathrm{CO}_{2}$ emission rate or explain variance in observed $\mathrm{CO}_{2}$ or isoprene emission rates. From 
446 Figure S6 in the Supporting Information, we do observe that the relation between isoprene 447 emission rates and three of the four HRV related markers are in the expected directions, that 448 is, human chemical emission of isoprene increases with increases in stress markers, though 449 not statistically significantly.

Prior studies have explored the relationship between cognitive tests and other factors 451 on human VOC emissions. Santos et al. ${ }^{55}$, with a study design that employed a cognitive test 452 to elicit stress in a cohort of 14 individuals, identified possible VOCs that are indicative of 453 stress. Their study identified benzaldehyde, ethyl acetate, and 2-propanol as indicators of 454 stress, compounds we found in the chamber at measurable levels in TD-GC-MS samples. 455 Santos et al. ${ }^{55}$ employed a prototype sampling device and analysis via GC-IMS. The GC-IMS 456 method is noted as subject to uncertainty in identification of compounds. We do not here 457 speculate concerning the possible relationship of these compounds to cognitive tasks, since 458 these compounds were not present in our calibration mixture. A recent study explored the relationship between temperature and human chemical 460 emissions, finding increases in ammonia emissions from humans with increasing 461 temperature. ${ }^{34}$ Our study design aimed to hold temperature constant; temperature averaged $46226.2 \pm 0.49 \circ \mathrm{C}($ mean \pm standard deviation). Given the known association between air 463 temperature and human $\mathrm{CO}_{2}$ emissions ${ }^{18}$ and recent observation of temperature dependence 464 on ammonia emissions, Figure 5 includes a regression of $\mathrm{CO}_{2}$ and isoprene emission rates as a 465 function of chamber air temperature, plotted as the natural log of emission rate vs. the inverse 466 of temperature. While slopes are in the expected direction (i.e., a negative slope, implying 467 higher temperature yields higher emission rate), the regression statistics indicate the 
relationship is not significant. A linear regression of emission rate vs. relative humidity also resulted in no significant correlation (Figure S6 of Supporting Information).

In this study, we limit our analysis to isoprene and $\mathrm{CO}_{2}$ as robust quantification of other VOCs is limited by the calibration standard and TD-GC-MS method applied to this chamber study. One further limitation is that our dynamic analysis of isoprene emission rates was constrained by time-integrated sampling in the chamber and inlet air. We believe the method for estimating per-person isoprene emission rates developed is robust; empty chamber isoprene emission rates were near zero while occupied chamber emission rates are in close agreement with prior estimates. However, there exists uncertainty associated with the initial conditions required for the solution, that is, the level of isoprene was assumed to be that of the baseline conditions at the start of an occupied chamber experiment. Sensitivity analyses indicate the solution is relatively insensitive to this input; model runs show a $20 \%$ change to the initial condition in the chamber results in a $\sim 5 \%$ change in the calculated isoprene emission rate. As discussed, surface adsorption/desorption of isoprene may impact the second occupied test of each day, though we believe the effect is likely small based on the high volatility of isoprene, our observation of near-zero isoprene emission rates in the unoccupied (baseline) test chamber, and counterbalanced design.

Subsequent chamber studies should employ additional analytical instrumentation, e.g., real-time chemical ionization mass spectrometry, to further elucidate the chemical complexity of human chemical emissions impacted by cognitive task. These instruments would greatly expand the classes of compounds that can be measured and improve robustness of source strength calculations with dynamic measurements. Future studies should also be conducted on larger sample populations, with different cognitive tasks, and examine potential for 
491 compounding interactions with other indoor environmental quality variables, e.g., varying

492 temperature and $\mathrm{RH}$ condition.

493 Supporting Information

494 The Supporting Information contains additional information concerning the test bed facility, 495 experimental conditions, details of cognitive tests used for the "stressed" activity,

496 characteristics of participants, and additional details of indicators of participant stress and

497 feedback. Further information is provided concerning the measurement of isoprene and

498 resulting calculation of isoprene emission rates. Additional data is reported from the

499 subjective feedback and pupillometry, analysis of $\mathrm{CO}_{2}$ regressions to determine $\mathrm{CO}_{2}$ emission

500 rates, and full results of regression of $\mathrm{CO}_{2}$ and isoprene emission rates with stress indicators

501 and chamber temperature and RH conditions.

\section{Author Contributions}

503 ETG led study design, analysis, and writing, AM contributed to study design, analysis and

504 writing, JL contributed to study design, analysis and data visualization, SS contributed to

505 study design, writing, and editing, AL contributed to data analysis and editing. All authors

506 have given approval to the final version of the manuscript.

\section{Acknowledgements}

508 This research was funded by the Republic of Singapore's National Research Foundation

509 through the SinBerBEST program. The research was carried out within the SinBerBEST

510 Testbed (http://sinberbest.berkeley.edu/content-page/testbed-facilities). We thank the

511 anonymous reviewers for their thoughtful and thorough comments that improved the paper. 


\section{References}

(1) Persily, A. K. Evaluating Building IAQ and Ventilation with Indoor Carbon Dioxide. ASHRAE Trans. 1997, 103, 193-204.

(2) Tang, X.; Misztal, P. K.; Nazaroff, W. W.; Goldstein, A. H. Volatile Organic Compound Emissions from Humans Indoors. Environ. Sci. Technol. 2016, 50 (23), 12686-12694. https://doi.org/10.1021/acs.est.6b04415.

(3) Bhangar, S.; Huffman, J. A.; Nazaroff, W. W. Size-Resolved Fluorescent Biological Aerosol Particle Concentrations and Occupant Emissions in a University Classroom. Indoor Air 2014, 24 (6), 604-617. https://doi.org/10.1111/ina.12111.

(4) Li, J.; Wan, M. P.; Schiavon, S.; Tham, K. W.; Zuraimi, S.; Xiong, J.; Fang, M.; Gall, E. Size-resolved Dynamics of Indoor and Outdoor Fluorescent Biological Aerosol Particles in a Bedroom: A Onemonth Case Study in Singapore. Indoor Air 2020, ina.12678. https://doi.org/10.1111/ina.12678.

(5) Weisel, C. P.; Fiedler, N.; Weschler, C. J.; Ohman-Strickland, P. A.; Mohan, K. R.; McNeil, K.; Space, D. R. Human Symptom Responses to Bioeffluents, Short-Chain Carbonyls/Acids, and Long-Chain Carbonyls in a Simulated Aircraft Cabin Environment. Indoor Air 2017, 27 (6), 11541167. https://doi.org/10.1111/ina.12392.

(6) Kruza, M.; Carslaw, N. How Do Breath and Skin Emissions Impact Indoor Air Chemistry? Indoor Air 2019, 29 (3), 369-379. https://doi.org/10.1111/ina.12539.

(7) ANSI/ASHRAE. Standard 62.1-2013 Ventilation for Acceptable Indoor Air Quality; American Society of Heating, Refrigerating and Air- Conditioning Engineers, Inc.: Atlanta, 2013.

(8) Seppänen, O.; Fisk, W. J.; Lei, Q. H. Ventilation and Performance in Office Work. Indoor Air 2006, 16 (1), 28-36. https://doi.org/10.1111/j.1600-0668.2005.00394.x.

(9) Mendell, M. J.; Heath, G. A. Do Indoor Pollutants and Thermal Conditions in Schools Influence Student Performance? A Critical Review of the Literature. Indoor Air 2005, 15 (1), 27-52. https://doi.org/10.1111/j.1600-0668.2004.00320.x.

(10) Satish, U.; Mendell, M. J.; Shekhar, K.; Hotchi, T.; Sullivan, D.; Streufert, S.; Fisk, W. J. Is $\mathrm{CO}_{2}$ an Indoor Pollutant? Direct Effects of Low-to-Moderate $\mathrm{CO}_{2}$ Concentrations on Human DecisionMaking Performance. Environ. Health Perspect. 2012, 120 (12), 1671-1677. https://doi.org/10.1289/ehp.1104789.

(11) Allen, J. G.; MacNaughton, P.; Satish, U.; Santanam, S.; Vallarino, J.; Spengler, J. D. Associations of Cognitive Function Scores with Carbon Dioxide, Ventilation, and Volatile Organic Compound Exposures in Office Workers: A Controlled Exposure Study of Green and Conventional Office Environments. Environ. Health Perspect. 2016, 124 (6), 805-812. https://doi.org/10.1289/ehp.1510037.

(12) Rodeheffer, C. D.; Chabal, S.; Clarke, J. M.; Fothergill, D. M. Acute Exposure to Low-to-Moderate Carbon Dioxide Levels and Submariner Decision Making. Aerosp. Med. Hum. Perform. 2018, 89 (6), 520-525. https://doi.org/10.3357/AMHP.5010.2018.

(13) Scully, R. R.; Basner, M.; Nasrini, J.; Lam, C.; Hermosillo, E.; Gur, R. C.; Moore, T.; Alexander, D. J.; Satish, U.; Ryder, V. E. Effects of Acute Exposures to Carbon Dioxide on Decision Making and Cognition in Astronaut-like Subjects. Npj Microgravity 2019, 5 (1), 1-15. https://doi.org/10.1038/s41526-019-0071-6.

(14) Zhang, X.; Wargocki, P.; Lian, Z. Human Responses to Carbon Dioxide, a Follow-up Study at Recommended Exposure Limits in Non-Industrial Environments. Build. Environ. 2016, 100, 162171. https://doi.org/10.1016/j.buildenv.2016.02.014.

(15) Zhang, X.; Wargocki, P.; Lian, Z.; Xie, J.; Liu, J. Responses to Human Bioeffluents at Levels Recommended by Ventilation Standards. Procedia Eng. 2017, 205, 609-614. https://doi.org/10.1016/j.proeng.2017.10.415. 
(16) Zhang, X.; Wargocki, P.; Lian, Z.; Thyregod, C. Effects of Exposure to Carbon Dioxide and Bioeffluents on Perceived Air Quality, Self-Assessed Acute Health Symptoms, and Cognitive Performance. Indoor Air 2017, 27 (1), 47-64. https://doi.org/10.1111/ina.12284.

(17) ASHRAE. Fundamentals Handbook.; American Society of Heating, Refrigerating and AirConditioning Engineers Inc: Atlanta: GA, 2013.

(18) Persily, A.; de Jonge, L. Carbon Dioxide Generation Rates for Building Occupants. Indoor Air 2017, 27 (5), 868-879. https://doi.org/10.1111/ina.12383.

(19) Black, A. E.; Prentice, A. M.; Coward, W. A. Use of Food Quotients to Predict Respiratory Quotients for the Doubly-Labelled Water Method of Measuring Energy Expenditure. Hum. Nutr. Clin. Nutr. 1986, 40 (5), 381-391.

(20) Perbellini, L.; Princivalle, A.; Cerpelloni, M.; Pasini, F.; Brugnone, F. Comparison of Breath, Blood and Urine Concentrations in the Biomonitoring of Environmental Exposure to 1,3-Butadiene, 2,5-Dimethylfuran, and Benzene. Int. Arch. Occup. Environ. Health 2003, 76 (6), 461-466. https://doi.org/10.1007/s00420-003-0436-7.

(21) Ligor, T.; Ligor, M.; Amann, A.; Ager, C.; Bachler, M.; Dzien, A.; Buszewski, B. The Analysis of Healthy Volunteers' Exhaled Breath by the Use of Solid-Phase Microextraction and GC-MS. J. Breath Res. 2008, 2 (4), 046006. https://doi.org/10.1088/1752-7155/2/4/046006.

(22) Mochalski, P.; King, J.; Klieber, M.; Unterkofler, K.; Hinterhuber, H.; Baumann, M.; Amann, A. Blood and Breath Levels of Selected Volatile Organic Compounds in Healthy Volunteers. The Analyst 2013, 138 (7), 2134-2145. https://doi.org/10.1039/c3an36756h.

(23) Mochalski, P.; King, J.; Unterkofler, K.; Hinterhuber, H.; Amann, A. Emission Rates of Selected Volatile Organic Compounds from Skin of Healthy Volunteers. J. Chromatogr. B 2014, 959, 6270. https://doi.org/10.1016/j.jchromb.2014.04.006.

(24) Chen, X.; Xu, F.; Wang, Y.; Pan, Y.; Lu, D.; Wang, P.; Ying, K.; Chen, E.; Zhang, W. A Study of the Volatile Organic Compounds Exhaled by Lung Cancer Cells in Vitro for Breath Diagnosis. Cancer 2007, 110 (4), 835-844. https://doi.org/10.1002/cncr.22844.

(25) Liu, S.; Li, R.; Wild, R. J.; Warneke, C.; de Gouw, J. A.; Brown, S. S.; Miller, S. L.; Luongo, J. C.; Jimenez, J. L.; Ziemann, P. J. Contribution of Human-Related Sources to Indoor Volatile Organic Compounds in a University Classroom. Indoor Air 2016, 26 (6), 925-938. https://doi.org/10.1111/ina.12272.

(26) Liu, S.; Thompson, S. L.; Stark, H.; Ziemann, P. J.; Jimenez, J. L. Gas-Phase Carboxylic Acids in a University Classroom: Abundance, Variability, and Sources. Environ. Sci. Technol. 2017, 51 (10), 5454-5463. https://doi.org/10.1021/acs.est.7b01358.

(27) Pagonis, D.; Price, D. J.; Algrim, L. B.; Day, D. A.; Handschy, A. V.; Stark, H.; Miller, S. L.; de Gouw, J.; Jimenez, J. L.; Ziemann, P. J. Time-Resolved Measurements of Indoor Chemical Emissions, Deposition, and Reactions in a University Art Museum. Environ. Sci. Technol. 2019, 53 (9), 47944802. https://doi.org/10.1021/acs.est.9b00276.

(28) Stönner, C.; Edtbauer, A.; Williams, J. Real-World Volatile Organic Compound Emission Rates from Seated Adults and Children for Use in Indoor Air Studies. Indoor Air 2018, 28 (1), 164-172. https://doi.org/10.1111/ina.12405.

(29) Sun, X.; He, J.; Yang, X. Human Breath as a Source of VOCs in the Built Environment, Part II: Concentration Levels, Emission Rates and Factor Analysis. Build. Environ. 2017, 123, 437-445. https://doi.org/10.1016/j.buildenv.2017.07.009.

(30) Schwarz, K.; Pizzini, A.; Arendacká, B.; Zerlauth, K.; Filipiak, W.; Schmid, A.; Dzien, A.; Neuner, S.; Lechleitner, M.; S Scholl-Bürgi; Miekisch, W.; Schubert, J.; Unterkofler, K.; Witkovský, V.; Gastl, G.; Amann, A. Breath Acetone-Aspects of Normal Physiology Related to Age and Gender as Determined in a PTR-MS Study. J. Breath Res. 2009, 3 (2), 027003. https://doi.org/10.1088/1752-7155/3/2/027003. 
607

608

609

610

611

612

613

614

615

616

617

618

619

620

621

622

623

624

625

626

627

628

629

630

631

632

633

634

635

636

637

638

639

640

641

642

643

644

645

646

647

648

649

650

651

652

653

654

(31) Herbig, J.; Beauchamp, J. Towards Standardization in the Analysis of Breath Gas Volatiles. J. Breath Res. 2014, 8 (3), 037101. https://doi.org/10.1088/1752-7155/8/3/037101.

(32) Amann, A.; Costello, B. de L.; Miekisch, W.; Schubert, J.; Buszewski, B.; Joachim Pleil; Ratcliffe, N.; Risby, T. The Human Volatilome: Volatile Organic Compounds (VOCs) in Exhaled Breath, Skin Emanations, Urine, Feces and Saliva. J. Breath Res. 2014, 8 (3), 034001. https://doi.org/10.1088/1752-7155/8/3/034001.

(33) Bekö, G.; Wargocki, P.; Wang, N.; Li, M.; Weschler, C. J.; Morrison, G.; Langer, S.; Ernle, L.; Licina, D.; Yang, S.; Zannoni, N.; Williams, J. The Indoor Chemical Human Emissions and Reactivity Project (ICHEAR): Overview of Experimental Methodology and Preliminary Results. Indoor Air n/a (n/a). https://doi.org/10.1111/ina.12687.

(34) Li, M.; Weschler, C. J.; Bekö, G.; Wargocki, P.; Lucic, G.; Williams, J. Human Ammonia Emission Rates under Various Indoor Environmental Conditions. Environ. Sci. Technol. 2020, 54 (9), 54195428. https://doi.org/10.1021/acs.est.0c00094.

(35) Wang, T. C. A Study of Bioeffluents in a College Classroom. ASHRAE Trans. 1975, 81 (1), 32-44.

(36) Williams, J.; Stönner, C.; Wicker, J.; Krauter, N.; Derstroff, B.; Bourtsoukidis, E.; Klüpfel, T.; Kramer, S. Cinema Audiences Reproducibly Vary the Chemical Composition of Air during Films, by Broadcasting Scene Specific Emissions on Breath. Sci. Rep. 2016, 6, 25464. https://doi.org/10.1038/srep25464.

(37) Stönner, C.; Edtbauer, A.; Derstroff, B.; Bourtsoukidis, E.; Klüpfel, T.; Wicker, J.; Williams, J. Proof of Concept Study: Testing Human Volatile Organic Compounds as Tools for Age Classification of Films. PLOS ONE 2018, 13 (10), e0203044. https://doi.org/10.1371/journal.pone.0203044.

(38) King, J.; Koc, H.; Unterkofler, K.; Mochalski, P.; Kupferthaler, A.; Teschl, G.; Teschl, S.; Hinterhuber, H.; Amann, A. Physiological Modeling of Isoprene Dynamics in Exhaled Breath. J. Theor. Biol. 2010, 267 (4), 626-637. https://doi.org/10.1016/j.jtbi.2010.09.028.

(39) Diamond, A. Executive Functions. Annu. Rev. Psychol. 2013, 64, 135-168. https://doi.org/10.1146/annurev-psych-113011-143750.

(40) Mueller, S. T.; Piper, B. J. The Psychology Experiment Building Language (PEBL) and PEBL Test Battery. J. Neurosci. Methods 2014, 222, 250-259.

https://doi.org/10.1016/j.jneumeth.2013.10.024.

(41) Kirschbaum, C.; Hellhammer, D. H. Salivary Cortisol in Psychoneuroendocrine Research: Recent Developments and Applications. Psychoneuroendocrinology 1994, 19 (4), 313-333. https://doi.org/10.1016/0306-4530(94)90013-2.

(42) Takai, N.; Yamaguchi, M.; Aragaki, T.; Eto, K.; Uchihashi, K.; Nishikawa, Y. Effect of Psychological Stress on the Salivary Cortisol and Amylase Levels in Healthy Young Adults. Arch. Oral Biol. 2004, 49 (12), 963-968. https://doi.org/10.1016/j.archoralbio.2004.06.007.

(43) Nejtek, V. A. High and Low Emotion Events Influence Emotional Stress Perceptions and Are Associated with Salivary Cortisol Response Changes in a Consecutive Stress Paradigm. Psychoneuroendocrinology 2002, 27 (3), 337-352. https://doi.org/10.1016/s03064530(01)00055-5.

(44) Pankow, J. F.; Luo, W.; Isabelle, L. M.; Bender, D. A.; Baker, R. J. Determination of a Wide Range of Volatile Organic Compounds in Ambient Air Using Multisorbent Adsorption/Thermal Desorption and Gas Chromatography/Mass Spectrometry. Anal. Chem. 1998, 70 (24), 52135221. https://doi.org/10.1021/ac980481t.

(45) Chin, K.; Laguerre, A.; Ramasubramanian, P.; Pleshakov, D.; Stephens, B.; Gall, E. T. Emerging Investigator Series: Primary Emissions, Ozone Reactivity, and Byproduct Emissions from Building Insulation Materials. Environ. Sci. Process. Impacts 2019, 21 (8), 1255-1267. https://doi.org/10.1039/C9EM00024K. 
(46) Nater, U. M.; Rohleder, N.; Schlotz, W.; Ehlert, U.; Kirschbaum, C. Determinants of the Diurnal Course of Salivary Alpha-Amylase. Psychoneuroendocrinology 2007, 32 (4), 392-401. https://doi.org/10.1016/j.psyneuen.2007.02.007.

(47) Dishman, R.; Nakamura, Y.; Garcia, M.; Thompson, R.; Dunn, A.; Blair, S. Heart Rate Variability, Trait Anxiety, and Perceived Stress among Physically Fit Men and Women. Int. J. Psychophysiol. 2000, 37 (2), 121-133.

(48) Hunt, K. J.; Saengsuwan, J. Changes in Heart Rate Variability with Respect to Exercise Intensity and Time during Treadmill Running. Biomed. Eng. Online 2018, 17 (1), 128. https://doi.org/10.1186/s12938-018-0561-x.

(49) Granholm, E.; Steinhauer, S. R. Pupillometric Measures of Cognitive and Emotional Processes. Int. J. Psychophysiol. 2004, 52 (1), 1-6. https://doi.org/10.1016/j.ijpsycho.2003.12.001.

(50) Salthammer, T. Very Volatile Organic Compounds: An Understudied Class of Indoor Air Pollutants. Indoor Air 2016, 26 (1), 25-38. https://doi.org/10.1111/ina.12173.

(51) Wang, C.; Collins, D. B.; Arata, C.; Goldstein, A. H.; Mattila, J. M.; Farmer, D. K.; Ampollini, L.; DeCarlo, P. F.; Novoselac, A.; Vance, M. E.; Nazaroff, W. W.; Abbatt, J. P. D. Surface Reservoirs Dominate Dynamic Gas-Surface Partitioning of Many Indoor Air Constituents. Sci. Adv. 2020, 6 (8), eaay8973. https://doi.org/10.1126/sciadv.aay8973.

(52) Stone, B. G.; Besse, T. J.; Duane, W. C.; Evans, C. D.; DeMaster, E. G. Effect of Regulating Cholesterol Biosynthesis on Breath Isoprene Excretion in Men. Lipids 1993, 28 (8), 705-708. https://doi.org/10.1007/BF02535990.

(53) Karl, T.; Prazeller, P.; Mayr, D.; Jordan, A.; Rieder, J.; Fall, R.; Lindinger, W. Human Breath Isoprene and Its Relation to Blood Cholesterol Levels: New Measurements and Modeling. J. Appl. Physiol. 2001, 91 (2), 762-770. https://doi.org/10.1152/jappl.2001.91.2.762.

(54) Maruyama, Y.; Kawano, A.; Okamoto, S.; Ando, T.; Ishitobi, Y.; Tanaka, Y.; Inoue, A.; Imanaga, J.; Kanehisa, M.; Higuma, H.; Ninomiya, T.; Tsuru, J.; Hanada, H.; Akiyoshi, J. Differences in Salivary Alpha-Amylase and Cortisol Responsiveness Following Exposure to Electrical Stimulation versus the Trier Social Stress Tests. PloS One 2012, 7 (7), e39375. https://doi.org/10.1371/journal.pone.0039375.

(55) Santos, P.; Roth, P.; Fernandes, J. M.; Fetter, V.; Vassilenko, V. Real Time Mental Stress Detection Through Breath Analysis. In Technological Innovation for Life Improvement; Camarinha-Matos, L. M., Farhadi, N., Lopes, F., Pereira, H., Eds.; IFIP Advances in Information and Communication Technology; Springer International Publishing: Cham, 2020; pp 403-410. https://doi.org/10.1007/978-3-030-45124-0_39. 